\title{
Transatlantica
}

Revue d'études américaines. American Studies Journal

1 | 2017

Morphing Bodies: Strategies of Embodiment in

Contemporary US Cultural Practices

Conférence « Liberating the Spirit of Women as an American Value » de Carol Moseley Braun, ancienne Sénatrice américaine et Ambassadrice de Nouvelle Zélande.

Université Sorbonne Nouvelle - Paris III, 16 novembre 2017

\section{Marine Dabon}

\section{(2) OpenEdition}

Édition électronique

URL : https://journals.openedition.org/transatlantica/8634

DOI : 10.4000/transatlantica.8634

ISSN : 1765-2766

Éditeur

Association française d'Etudes Américaines (AFEA)

Référence électronique

Marine Dabon, «Conférence « Liberating the Spirit of Women as an American Value » de Carol Moseley Braun, ancienne Sénatrice américaine et Ambassadrice de Nouvelle Zélande. »,

Transatlantica [En ligne], 1 | 2017, mis en ligne le 03 décembre 2018, consulté le 24 mai 2021. URL http://journals.openedition.org/transatlantica/8634; DOI : https://doi.org/10.4000/transatlantica 8634

Ce document a été généré automatiquement le 24 mai 2021.

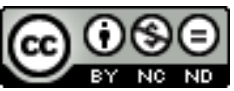

Transatlantica - Revue d'études américaines est mise à disposition selon les termes de la licence Creative Commons Attribution - Pas d'Utilisation Commerciale - Pas de Modification 4.0 International. 


\section{Conférence « Liberating the Spirit of Women as an American Value » de Carol Moseley Braun, ancienne Sénatrice américaine et Ambassadrice de Nouvelle Zélande.}

Université Sorbonne Nouvelle - Paris III, 16 novembre 2017

Marine Dabon

1 L'université Sorbonne Nouvelle a reçu, le 16 novembre 2017, Carol Moseley Braun, ancienne Sénatrice et Ambassadrice américaine. Cette conférence, intitulée "Liberating the Spirit of Women as an American Value " a été organisée par Hélène Quanquin et Christine Zumello du Centre de Recherche sur l'Amérique de Nord (CRAN) au sein de l'équipe de recherche CREW (EA-4399). Cette conférence a porté sur la place des femmes dans la société et la vie politique américaine et s'inscrit dans un contexte politique qui amène les femmes à se questionner sur leur rôle au sein de la société, en particulier depuis l'élection de Donald Trump à la présidence des Etats-Unis en novembre 2016. Les organisatrices n'ont pas manqué de souligner l'ironie de la tenue de cette conférence le même jour que la journée d'étude « Donald Trump et la politique étrangère des Etats-Unis : vers quel (dés)ordre mondial ?».

2 Après avoir accueilli et remercié chaleureusement Carol Moseley Braun de sa visite, le Président de l'université Sorbonne Nouvelle a laissé la parole à Hélène Quanquin pour une brève biographie. Carol Moseley Braun fut la première femme afro-américaine élue au Sénat. Après un mandat de Sénatrice de l'Illinois de 1993 à 1999, elle fut Ambassadrice des Etats-Unis en Nouvelle Zélande et à Samoa de 1999 à 2001, et candidate aux primaires démocrates pour les élections présidentielles en 2004.

3 Christine Zumello a remercié à son tour l'Ambassadrice, en la qualifiant d'« inspiration, à la fois pour les femmes et pour les hommes ». 


\section{La libération de l'esprit : responsabilité individuelle au service de la société}

4 L'auditoire venu écouter l'ancienne Sénatrice a eu le plaisir de l'entendre commencer son discours en français - langue qu'elle étudie depuis plusieurs années déjà. Elle a ensuite préféré poursuivre en anglais.

5 Carol Moseley Braun nous a d'abord dépeint une enfance passée à Chicago, élevée par des parents ayant grandi dans une société très marquée par la ségrégation. Elle nous a parlé de l'influence qu'ont eue Martin Luther King et la lutte pour les droits civiques dans la construction de son identité de femme afro-américaine. Elle a côtoyé Dr. King et a même manifesté à ses côtés. Elle s'est mariée avec un homme blanc, consciente que cela n'aurait pas pu être possible sans le travail du leader du mouvement pour les droits civiques. Sa carrière l'a menée de la législature de l'Illinois au Sénat en passant par la Nouvelle Zélande et Samoa. Après une carrière en politique, elle a lancé sa marque de boissons bios, "Ambassador Organics", se qualifiant dorénavant de politicienne en convalescence ( recovering politician »). Elle enseigne également les sciences politiques à l'université Northwestern de Chicago.

6 La notion de "rêve américain" est prédominante dans son discours. Il est de la responsabilité de chaque citoyen de faire en sorte de laisser aux générations futures une société plus avancée. Cette évolution de la société passe par la libération de l'esprit humain («the liberation of the human spirit»), thème central du discours de Carol Moseley Braun. Des facteurs comme le genre ou le handicap ne devraient plus être des obstacles à la réussite et au bonheur de chacun. Chaque personne - homme ou femme a un rôle à jouer dans cette évolution sociétale. Elle affirme ainsi que nos actions définissent le présent et créeront le futur (« will shape today and create tomorrow»). Ce sont des actions individuelles, parfois discrètes et anonymes, qui contribuent à la prospérité culturelle, économique et sociale de la communauté.

7 L'ambassadrice a illustré son propos par l'exemple de Charley, un esclave noir qui a fui le Missouri dans les années 1850 en traversant le fleuve Mississippi à la nage et qui fut secouru par le Dr. William Eells à Quincy, dans l'Illinois. Poursuivi par la police, Dr. Eells tenta de fuir avec Charley. Celui-ci sauta du chariot et réussi à s'enfuir. Malheureusement, Dr. Eells fut arrêté, traduit en justice et condamné par le Juge Douglas à payer $\$ 400$. Cette affaire eut un écho jusqu'à la Cour Suprême des Etats-Unis et fut au cœur du débat entre Abraham Lincoln et Stephen Douglas, tous deux candidats aux élections sénatoriales pour l'état de l'Illinois. Si Lincoln remporta le débat, ce fut Douglas qui gagna les élections et vota le "Kansas-Nebraska Act " - le même Douglas dont Carol Moseley Braun devait occuper le bureau 100 ans plus tard, ainsi qu'elle le fit remarquer non sans ironie. Malgré la victoire de Douglas et la condamnation du Dr. Eels, cette affaire permit de centrer les débats politiques sur la question de l'esclavage. Soucieuse d'honorer la mémoire de ces événements, la Sénatrice fut également amenée à voter pour la protection des chemins de fer clandestins ("Underground Railroads ») qui aidèrent de nombreux esclaves à fuir. L'ambassadrice tenait à montrer que par son sacrifice, William Eells a œuvré pour l'abolition de l'esclavage, et l'évolution de la société américaine. C'est également grâce à lui que Carol Moseley Braun, descendante d'esclaves, a pu occuper la fonction de Sénatrice. 
8 Lorsqu'elle fut Ambassadrice des Etats-Unis en Nouvelle Zélande, elle fut nommée membre honorifique d'une tribu Maori. Leur philosophie sur le temps l'a beaucoup inspirée. Pour la tribu Te Atiawa, le futur est derrière nous car nous ne pouvons pas le voir. Il demeure mystérieux et inconnu. En revanche, le passé se tient devant nous, afin que nous puissions en tirer les leçons. Le futur est la conséquence de nos actions présentes et de notre analyse des actions passées.

9 Abigail Adams, épouse de John Adams - deuxième Président des Etats-Unis, fut très active pour l'égalité hommes-femmes. Elle montra les contradictions soulevées par la Révolution américaine - révolution qui visait à obtenir des libertés fondamentales, mais qui ne devaient pas s'appliquer aux femmes, aux Indiens et aux hommes blancs les moins fortunés. Citant les mots d'Abigail Adams, Carol Moseley Braun prononça : «I have sometimes been ready to think that the passion for liberty cannot be equally strong in the breasts of those who have been accustomed to deprive their fellow creatures of theirs ". L'égalité des sexes est, pour l'ancienne Sénatrice, toujours au cœur du débat dans la société américaine.

10 L'ambassadrice est convaincue que nous sommes tous responsables de la direction que prend la société - que ce soit vers la tolérance, ou au contraire vers le rejet. Le racisme a reculé ces dernières années, et le sexisme commence à être dénoncé. C'est donc à chaque citoyen de poursuivre dans cette direction. Martin Luther King n'avait que 25 ans lorsqu'il prit la tête du mouvement pacifiste pour les droits civiques. L'activisme politique et la volonté de transformer positivement la société doivent concerner les plus jeunes.

11 Carol Moseley Braun partagea une anecdote personnelle, qui fit sourire l'auditoire, malgré le contexte politique dans lequel l'histoire eut lieu. Lorsqu'elle était enfant, elle était en voyage avec ses frères et ses parents vers la ferme familiale en Alabama lorsque, arrivés dans une gare ferroviaire, son frère demanda à sa mère s'il pouvait boire à la fontaine, réservée aux noirs. Sa mère refusa et le pria d'attendre d'être arrivé. Le frère de Carol Moseley Braun fit un caprice, ne comprenant pas pourquoi il n'avait pas le droit de boire l'eau de la "colored fountain», pensant qu'un arc-en-ciel en jaillirait. La Sénatrice souhaitait par ce biais montrer l'absurdité de la ségrégation raciale pour un enfant dans les années 1950. Ce sont les sacrifices personnels qui ont permis de changer les mentalités et les lois.

12 Le progrès n'est pas linéaire. Les décisions que nous prenons ne produiront pas forcément le résultat que nous attendons. Mais il est nécessaire que l'intérêt collectif passe avant les ambitions personnelles. Le pouvoir de transformer positivement la société repose entre dans les mains des citoyens. Le progrès est fait d'une succession d'actions.

13 Les femmes ont obtenu le droit de vote en 1920 aux Etats-Unis. L'Etat du Tennessee est le dernier à avoir accordé ce droit. La veille du vote, Harry Burn, député Républicain au départ opposé au 19ème Amendement, reçut une lettre de sa mère, lui demandant d'être « un bon garçon » et de prendre la bonne décision. Harry Burn changea d'avis et vota en faveur de l'Amendement, garantissant ainsi le droit de vote aux femmes. Nous ne connaissons à ce jour pas ce qui a motivé la mère d'Harry Burn à écrire cette lettre qui changea l'issue du vote. Elle n'imaginait sans doute pas que sa lettre aurait un tel impact dans l'histoire américaine. 
Le monde que nous laisserons sera meilleur que celui dont nous avons hérité. Il est du devoir de chacun d'y contribuer, par des actions individuelles au service du bien collectif. Carol Moseley Braun a conclu son discours avec un poème néo-zélandais sur la responsabilité individuelle, avec l'idée qu'une multitude de micro-actions peut transformer les mœurs.

\section{Questions}

Les questions posées à l'ambassadrice Moseley Braun portèrent essentiellement sur sa carrière en tant que Sénatrice. Elle voyait son rôle de Sénatrice comme une manière d'exprimer ses opinions, son activisme politique. Toujours dans l'optique que chaque décision a un impact sur la société, elle a essayé tout au long de sa carrière d'allier son activisme politique à ses diverses fonctions. Elle pense d'ailleurs que, malgré l'élection de Donald Trump, les Démocrates et les Républicains ont le pouvoir de travailler pour le bien de la société américaine.

Des questions plus personnelles furent ensuite posées, sur ses motivations et ses croyances personnelles. Elle expliqua que, malgré les difficultés et le traitement souvent négatif qu'elle a reçu tout au long de sa carrière, elle n'a jamais baissé les bras car ses convictions l'ont toujours poussée à continuer d'avancer.

Enfin, les questions se concentrèrent sur la place des femmes dans la vie politique américaine. Carol Moseley Braun est convaincue que le plus grand obstacle à l'égalité des sexes en politique est la mentalité d'une société encore profondément patriarcale. Lorsqu'elle était Sénatrice, les élues se réunissaient de manière officieuse et bipartisane afin de travailler ensemble. Depuis l'élection de Donald Trump, de plus en plus de femmes se présentent à des élections à différentes échelles, locales ou nationales. Cela montre que la société est en train d'évoluer, ce dont se réjouit la Sénatrice.

\section{RÉSUMÉS}

Ce compte-rendu résume le discours de Carol Moseley Braun, ancienne Sénatrice et Ambassadrice américaine, prononcé dans le cadre de la conférence intitulée «Liberating the Spirit of Women as an American Value ", organisée par Hélène Quanquin et Christine Zumello le 16 novembre 2017 à l'université Sorbonne Nouvelle.

This review is a summary of former American Senator and Ambassador Carol Moseley Braun's speech during a conference entitled "Liberating the Spirit of Women as an American Value ", organized by Hélène Quanquin and Christine Zumello and held on November $16^{\text {th }}$ in université Sorbonne Nouvelle. 
INDEX

Thèmes: Actualité de la recherche

Keywords : American politics, gender, activism, equality

Mots-clés : Politique américaine, genre, activisme, égalité

\section{AUTEUR}

MARINE DABON

Université Sorbonne Nouvelle - Paris III 